\title{
The Metaphysical Interpretation of Logical Truth
}

\author{
TUOMAS E. TAHKO (www.ttahko.net)
}

Theoretical Philosophy, University of Helsinki

In Penelope Rush (ed.), The Metaphysics of Logic: Logical Realism, Logical Anti-Realism and All Things In Between (Cambridge University Press, 2014), pp. 233-48.

\section{Two senses of logical truth}

The notion of logical truth has a wide variety of different uses, hence it is not surprising that it can be interpreted in different ways. In this chapter I will focus on one of them - what I call the metaphysical interpretation. A more precise formulation of this interpretation will be put forward in what follows, but I wish to say something about my motivation first. Part of my interest concerns the origin or ground of logic and logical truth, i.e., whether logic is grounded in how the world is or how we (or our minds) see the world. ${ }^{1}$ However, this is not my topic here. Rather, I will assume that logic is grounded in how the world is - a type of realism about logic - and examine the status of logical truth from the point of view of logical realism. The upshot is an interpretation of logical truth that is of special interest to metaphysicians. $^{2}$

My starting point is the apparent difference between what we might call absolute truth and

1 For a recent discussion on this topic, see Sher (2011), who examines the idea that logic is grounded either in the mind or in the world, and defends that it is grounded in both - hence logic has a dual nature. See also the opening chapter of this volume.

2 See Chateaubriand Filho $(2001,2005)$ for a version of the metaphysical interpretation of logical truth partly similar to mine. 
truth in a model, following Davidson (1973). The notion of absolute truth is familiar from Tarski's T-schema: 'Snow is white' is true if and only if snow is white - in the world and absolutely. Instead of being a property of sentences as absolute truth appears to be, truth in a model, that is relative truth, is evaluated in terms of the relation between sentences and models. ${ }^{3}$ Davidson suggested that philosophy of language should be interested in absolute truth exactly because relative truth does not yield T-schemas, but I am not concerned with this proposal here. ${ }^{4}$

To clarify, relative truth is an understanding of logical truth in terms of truth in all models. One can be a realist or an anti-realist about the models, hence about logical truth. But there are choices to be made even if one is realist about the models, as the models can be understood interpretationally or representationally, along the lines suggested by John Etchemendy (1990). We will discuss the difference between these views in the next section, but ultimately none of these alternatives are expressive of the metaphysical interpretation of logical truth. Instead, we need a way to express absolute truth, which is not possible without spelling out the correspondence intuition, to be discussed in a moment.

Given the topic of this chapter, one might expect that Michael Dummett's view would be discussed, or at least used as a foil, but I prefer not to dwell on Dummett. The primary reason for this is that Dummett's methodology is entirely opposite to the one that I use. Here is a summary of Dummett's method:

My contention is that all these metaphysical issues [questions about truth, time etc.]

3 'Models' are to be interpreted in a wide sense: they may for instance be interpretations, possible worlds or valuations. We will return to this ambiguity concerning 'model' below.

4 I should mention that I will omit discussion of Carnap and Quine on logical truth, as their debate is not directly relevant for my purposes. However, see Shapiro (2000) for an interesting discussion of Quine on logical truth. 
turn on questions about the correct meaning-theory for our language. We must not try to resolve the metaphysical questions first, and then construct a meaning-theory in the light of the answers. We should investigate how our language actually functions, and how we can construct a workable systematic description of how it functions; the answers to those questions will then determine the answers to the metaphysical ones. (Dummett 1991: 338.)

Since I am analyzing logical truth from a realist, metaphysical point of view, Dummett's methodology is obviously not going to do the trick. In my view, there is a bona fide discipline of metaphysics and I am interested in finding a use for logical truth within that discipline. I doubt there is enough initial common ground to fruitfully engage with Dummett.

Let me briefly return to Davidson and Tarski before proceeding. When considering the distinction between absolute and relative truth, an initial point of interest is the characterization of absolute truth by the T-schema. One question that emerges is the connection between the T-schema and metaphysics. A likely approach is to explicate this connection in terms of correspondence. However, at least according to one reading, Tarski (1944) considered truth understood as a semantic concept to be independent of any considerations regarding what sentences actually describe, that is, independent of issues concerning correspondence with the world. Indeed, the T-schema is now rarely considered to play a crucial role in correspondence theories of truth, despite the appearance of a correspondence relation between sentences and the world. ${ }^{5}$ Yet, Tarski’s (ibid., 342-3) initial

5 Furthermore, the idea that the T-schema or the correspondence theory are somehow expressive of realism has been forcefully disputed. See for instance Morris (2005) for a case against the connection between realism and correspondence; in fact Morris argues that correspondence theorists should be idealists. See also Gómez-Torrente (2009) for a discussion about Tarski's ideas on logical consequence as well as on Etchemendy's critique of Tarski's model-theoretic account. 
considerations on the meaning of the term 'true' explicitly take into account an 'Aristotelian' conception of truth, where correspondence with the world is central. Davidson (1973: 70) as well seems to have some sympathy for the idea that an absolute theory of truth is, in some sense, a 'correspondence theory' of truth, although he insists that the entities that would act as truthmakers here are 'nothing like facts or states of affairs', but sequences (which make true open sentences).

I will not aim to settle the status of the correspondence theory here, but it will be necessary to discuss it in some more detail. I suggest adopting an understanding of the correspondence relation which is neutral in terms of our theory of truth. It is this type of weak correspondence intuition that I believe central to the metaphysical interpretation of logical truth. But it should be stressed that the correspondence intuition itself is not necessarily expressive of realism (Daly 2005: 96-7). For instance, Chris Daly’s suggested definition of the intuition is simply that a proposition is true if and only if things are as the proposition says they are. Daly explains the neutrality of (his version of) the correspondence intuition as follows: ${ }^{6}$

Consider the coherence theorist. He may consistently say 'If $<p>$ is true, it has a truthmaker. $<p>$ corresponds to a state of affairs, namely the state of affairs which consists of a relation of coherence holding between $<p>$ and the other members of a maximal set of propositions'. Consider the pragmatist. He may consistently say, 'If $<p>$ is true, it has a truthmaker. $<p>$ corresponds to a state of affairs, namely the state of affairs of $<p>$ 's having the property of being useful to believe'. It is controversial whether there exist states of affairs. Let that pass. My point here is that the coherence theory and the pragmatic theory are each compatible with the admission of states of

6 The angled brackets describe a proposition, following Horwich (1998). 
affairs. Furthermore, each of these theories is compatible with the admission of states of affairs standing in a correspondence relation to truths. (Daly 2005: 97.)

A neutral version of the correspondence intuition is desirable because I do not want to rule out the possibility of different approaches to truth, despite assuming realism in the present context. A central appeal of the correspondence intuition is, I suggest, its wide applicability. However, a slightly better formulation than Daly’s can be found by following Paolo Crivelli (2004), who interprets Aristotle as an early proponent of the correspondence theory. Crivelli defines correspondence-as-isomorphism as follows: 'a theory of truth is a correspondence theory of truth just in case it takes the truth of a belief, or assertion, to consist in its being isomorphic with reality' (Crivelli 2004: 23). ${ }^{7}$ This type of view, which Crivelli ascribes to Aristotle, is expressive of the correspondence intuition, but avoids mention of propositions, or indeed states of affairs. ${ }^{8}$ Hence, we may define the correspondence intuition as follows:

(CI) A belief, or an assertion, is true if and only if its content is isomorphic with reality.

This formulation preserves Daly's idea. 'Reality' in CI may consist, say, of what it is useful to believe, as the pragmatist would have it, so neutrality is preserved. If we accept that CI is neutral in terms of different theories of truth, then we can characterize the issue at hand

7 Crivelli also defines a stricter sense of correspondence, which can be found in Aristotle. But sometimes Aristotle's view on truth is also considered as a precursor to deflationism about truth, so we shouldn't put too much weight on the historical case. For a more historically inclined discussion, see Paul Thom's chapter in this volume.

8 Admittedly, once we explicate isomorphism, reference to propositions, states of affairs or something of the sort could easily re-emerge. This shouldn't worry us too much, because it is likely that we want a structured mapping from something to reality. The reason to opt for isomorphism here is merely to keep the door open for one's preferred (structured) ontology. 
as follows. There is an apparent and important difference between truth understood along the lines of CI, and truth understood as a relation between sentences and models. I take this to be at the core of Davidson's original puzzle concerning absolute and relative truth. We ought to inquire into these two senses of truth before we give a full account of logical truth. This is exactly what I propose to do, arguing that the metaphysical interpretation of logical truth must respect CI.

Tarski and the model-theoretic approach may have made it possible to talk about logical truth in a manner seemingly independent of metaphysical considerations, but important questions about the metaphysical status of logical truth and the interpretation of models remain. One thing that makes this problem topical is the recent interest in logical pluralism, or pluralism about logical truth (e.g., Beall and Restall 2006). In the second section I will assess the metaphysical status of the notion of logical truth with regard to the two senses of truth familiar from Davidson. The third section takes up the issue of interpreting logical truth in terms of possible worlds and contains a case study of the law of non-contradiction. A brief discussion of logical pluralism will take place in the fourth section, before the concluding remarks.

\section{Reconciling the two senses of truth}

Can we reconcile the two senses of truth familiar from Davidson, the absolute and the relative? As Etchemendy (1990: 13) notes, the obvious way to attempt this would be in terms of generalization: if absolute truth is a monadic predicate of the form ' $\mathrm{x}$ is true', then it may be helpful to analyze it in terms of a relational predicate of the form ' $\mathrm{x}$ is true in $\mathrm{y}$ '. For instance ' $x$ is a brother' could be analyzed by first analyzing ' $x$ is a brother of $y$ ', thus using 
the generalized concept of brotherhood. However, this does not apply to truth: '[C]learly the monadic concept of truth, the concept we ordinarily employ, is no generalization of any of the various relational concepts. A sentence can be true in some model, yet not be true; a sentence can be true, yet not be true in all models' (ibid., 14). Accordingly, generalization will not help in reconciling the two senses of truth.

Another alternative that Etchemendy considers is to interpret absolute truth as a specification of truth in a model, namely, absolute truth could be considered equivalent to truth in the right model, the model that corresponds with the world. This maintains the correspondence intuition expressed by CI above, but note that 'correspondence with the world' already suggests a realist theory of truth, so the neutrality of the formulation is in question. ${ }^{9}$

However, there are good reasons to think that the notion of 'model' is not entirely appropriate when discussing absolute truth, as it is closely associated with relative truth. Hence, interesting as Etchemendy’s characterization may be, it is unlikely to result in a metaphysical account of logical truth. Still, Etchemendy's account may help pinpoint the issue; consider the following passage:

Once we have specified the class of models, our definition of truth in a model is guided by straightforward semantic intuitions, intuitions about the influence of the world on truth values of sentences in our language. Our criterion here is simple: a sentence is to be true in a model if and only if it would have been true had the model been accurate-that is, had the world actually been as depicted by that model.

9 Note that the question concerning which model is 'right' is not, strictly speaking, a question for the logician. For instance, as Burgess (1999: 82) notes, it is the metaphysician's task to determine the correct modal logic, as this depends on our understanding of (metaphysical) modality. In contrast, the question about the 'right' sense of logical validity remains in the realm of logic. 
(Etchemendy 1990: 24.)

There is an important requirement in the passage above, namely, it must be the case that the model could have been true. How do we interpret the modality in effect here? If we understand it as saying that it must be the case that the world could have turned out to be like the model depicts, then this supports the case for a metaphysical interpretation of logical truth, for it introduces as a requirement for the notion of 'model' that it is a possible representation of the world. This representational approach, or 'representational semantics' can be contrasted with ‘interpretational semantics’, which Etchemendy discusses later on:

[I]n an interpretational semantics, our class of models is determined by the chosen satisfaction domains; our definition of truth in a model is a simple variant of satisfaction. (Etchemendy 1990: 50.)

Etchemendy claims that the Tarskian conception of model-theoretic semantics is of the 'interpretational' kind, although his interpretation of Tarski can certainly be questioned (e.g. Gómez-Torrente 1999). But I do not wish to enter the debate about Tarski or interpretational semantics. According to Etchemendy, in the representational approach models must represent 'genuinely possible configurations' of the world, and I am interested in the correct understanding of these possible configurations (cf. Etchemendy 1990: 60). However, instead of developing Etchemendy's representational account, I will propose a pre-theoretic account of absolute truth, which aligns nicely with Etchemendy's analysis. The biggest complication is the interpretation of the modal content in Etchemendy's picture; we will need to return to this issue later (in the next section). 
What I propose to draw from Etchemendy is that once we have specified the class of 'genuinely possible configurations', we can define relative truth according to Etchemendy's suggestion. In this regard, my analysis will not follow that of Etchemendy's, as the case for absolute truth will come before Etchemendy's account. Etchemendy's representational approach notwithstanding, the notion of 'model' is not ideal for this task, as it is strongly reminiscent of relative truth. ${ }^{10}$

Instead of 'models', I propose to resort to talk of 'possible worlds'. What I have in mind is interpreting possible worlds as metaphysical possibilities. This is, of course, somewhat controversial, but as we will see, there are reasons to think that only metaphysical modality is fitting for the task. In any case, more needs to be said about how the space of metaphysical possibilities is restricted. We will return to this in the next section.

We are now in the position to define a provisional sense of logical truth which I propose to call metaphysical:

(ML) A sentence is logically true if and only if it is true in every genuinely possible configuration of the world.

ML leaves open the criteria for a 'genuinely possible configuration of the world'. But it does preserve CI and it provides us - via the possible worlds jargon - a 'metaphysician friendly' interface to the notion of logical truth. It is time to see if we can actually work with that interface.

10 It has been suggested to me (by Penny Rush) that relative truth may be problematic because of its underlying metaphysical commitment to relativism, rather than not being up to the job of giving a metaphysical interpretation of logical truth at all. This may indeed be the case. I have attempted to preserve ontological neutrality while at the same time making it clear that I am presently only interested in putting forward a realist interpretation of logical truth. But I will set this issue aside for now, whether or not it is possible to combine relative truth and realism. 


\section{Genuinely possible configurations and the case of the law of non-contradiction}

The puzzle can now be expressed in the following form: What sort of criteria can be established to evaluate whether a given possible world is a genuinely possible configuration of the world, i.e., could have turned out to correspond with the actual world? Let me approach the problem with a case study. Take, arguably, one of the most fundamental laws of logic, the law of non-contradiction (LNC). When I say that the law of non-contradiction is true in the 'metaphysical sense', I mean that LNC is true in the sense of absolute truth, i.e., it is a genuine constraint on the structure of reality. The metaphysical formulation of LNC takes a form familiar from Aristotle (Metaphysics 1005b19-20), although my proposed formulation is somewhat weaker, defined as follows:

(LNC) The same attribute cannot at the same time belong and not belong to the same subject in the same respect and in the same domain.

The above formulation differs from Aristotle's only with regard to the qualification regarding 'the same domain' - here the domain is the set of genuinely possible configurations of the world. How do we know whether LNC is true in this sense? I have previously argued (Tahko 2009) that we do have a good case for the truth of LNC in the metaphysical sense the primary opponent here is Graham Priest (e.g., 2006, 2006a). ${ }^{11}$ I will not repeat my arguments here, but it may be noted that this is not strictly a question for logic. For instance,

11 See also Berto 2008 for an attempt to formulate a (metaphysical) version of LNC which even the dialetheist must accept. Berto's idea, to which I am sympathetic, is that LNC may be understood as a principle regarding structured exclusion relations (between properties, states of affairs, etc.), and the world is determinate insofar as it conforms to this principle. 
Priest's most celebrated arguments in favor of true contradictions (in the metaphysical sense) concern the nature of change and specifically motion, the paradoxical nature of which is supposedly demonstrated by Zeno’s well known paradoxes. Although these paradoxes can quite easily be tackled by mathematical means, the relevant question is whether change indeed is paraconsistent. ${ }^{12}$ The answer to this question requires both metaphysical and empirical inquiry. I will return to this point briefly below, but first I wish to say something about the methodology of logical-cum-metaphysical inquiry.

In terms of ML, demonstrating the falsity of LNC would first require a genuinely possible configuration of the world where LNC fails. That is, it is not enough that we have a model where LNC is not true, such as paraconsistent logic, but we would also need to have some good reasons to think that the world could have been arranged in such a way that the implications of the metaphysical interpretation of LNC do not follow. This point deserves to be emphasized, for it would be much easier to show that a paraconsistent model can be useful in modelling certain phenomena, or interpreted in such a way that it is compatible with all the empirical data. But what is required here is that LNC, fully interpreted in the metaphysical sense, can be shown to fail.

Note that we may also ask whether LNC is necessary, i.e., are there any possible worlds in which LNC does not hold - even if we did have a good case for its truth in the actual world? In fact, this is the question we should begin with, since if LNC is necessary, then it could not fail in the actual world either. However, it is not clear how we could settle this question conclusively, given that we are dealing with the metaphysical interpretation of LNC. Moreover, I do think that there could (in an epistemic sense) be possible worlds in which LNC fails, and hence I take the debate about LNC seriously. Yet, I am uncertain about

12 For discussion regarding Zeno’s paradoxes, see for instance Sainsbury 2009: Ch. 1. 
whether such a paraconsistent possible world is in fact a genuinely possible configuration, as I will go on to explain. ${ }^{13}$ In any case, if a possible world in which LNC is not true were genuinely possible, then LNC would obviously not be necessary. This should be relatively uncontroversial, but I should finally say something more about 'genuine possibility'.

As was mentioned in the previous section, there are reasons to understand genuine possibility in terms of metaphysical possibility, as only metaphysical modality could secure the correspondence between a possible world and the structure of reality - this is also what CI requires. The relevant modal space must consist of all possible configurations of the world and only them. Logical modality cannot do the job because it is not sufficiently restrictive. This can be demonstrated with any traditional example of a metaphysical, a posteriori necessity, such as gold being the element with atomic number 79. Assuming that it is indeed metaphysically necessary that gold is the element with atomic number 79 , we must be able to accommodate the fact that gold failing to be the element with atomic number 79 is nevertheless logically possible. But since we are interested in genuinely possible configurations of the world, we ought to rule out metaphysically impossible worlds, such as the world in which gold fails to be the element with atomic number 79. The upshot is that if we accept the familiar story about metaphysical a posteriori necessities of this type, then there are necessary constraints for the structure of reality which logical necessity does not capture. $^{14}$

13 It is worth pointing out here that in my proposed construal, the distinction between absolute truth and truth in a model is not quite so striking for dialetheists. The idea, which I owe to Francesco Berto, is that the world cannot be a model, because it contains everything, and there's no domain of everything, on pain of Cantor's paradox. The result is that something can be a logical truth in sense of being true in all models, without being true in the absolute sense, for the world is not a model. My proposed treatment of this issue proceeds by understanding absolute truth in terms of metaphysical modality, but the dialetheist could, in principle, endorse paraconsistent set theory and posit that absolute truth is just truth in the world-model - the model whose domain is the world.

14 I should add that cashing out these constraints is, I think, a much more complicated affair than the traditional Kripke-Putnam approach to metaphysical a posteriori necessities suggests. 
The only other viable alternative in addition to metaphysical and logical modality is conceptual modality, i.e., necessity in virtue of the definitions of concepts. Nomological modality is already too restrictive, as we sometimes need to consider configurations of the world that are nomologically impossible but at least may be genuinely possible (e.g., superluminal travel). However, conceptual modality is too liberal, quite like logical modality, as it also accommodates configurations of the world which are not genuinely possible, such as violations of the familiar examples of metaphysical a posteriori necessities. If we accept these examples, then neither definitions of concepts or laws of logic rule out things like gold failing to be the element with atomic number 79. Accordingly, if one accepts that there are metaphysical necessities that are not also conceptually and logically necessary - something that most metaphysicians would accept - the only available interpretation of genuine possibility is in terms of metaphysical possibility.

There is, however, a way to understand logical modality which may do a better job in capturing the relevant sense of logical truth. This type of understanding has been proposed by Scott Shalkowski, who suggests that 'logical necessities might be explained as those propositions true in virtue of the natures of every situation or every object and property, thus preserving the idea that logic is the most general science' (Shalkowski 2004: 79). On the face of it, this suggestion respects the criteria for genuine possibility. According to this approach, logical modality concerns the most general (metaphysical) truths, such as the law of noncontradiction when it is considered as a metaphysical principle (as in Tahko 2009). In this view, logical relations reflect the relations of individuals, properties, and states of affairs rather than mere logical concepts. Indeed, this understanding effectively equates metaphysical and logical modality. The idea is that the purpose of logic is to describe the structure of reality and so it is 'the most general science'. As Shalkowski (ibid., 81) notes, denying the truth of LNC would, in terms of this understanding, amount to a genuine 
metaphysical attitude instead of, say, the fairly trivial point that a model in which the law does not hold can be constructed.

Do we have any means to settle the status of LNC in the suggested sense? A simple appeal to its universal applicability may not do the trick, but the burden of proof is arguably on those who would deny LNC. One might even attempt to distill a more general formula from this: logical principles - which are presumably reached by a priori means - are prima facie metaphysically necessary principles. They may be challenged and sometimes falsified even by empirical means, but merely the fact that we can formulate models in which they do not hold is not enough to challenge their truth; it will also have to be demonstrated that there are possible worlds which constitute genuinely possible configurations of the world. However, this approach seems biased towards historically prior logical principles, the ones that were formulated first. It is not implausible that the reason why they were formulated first is because they are indeed the best candidates for metaphysically necessary principles: for Aristotle, the law of non-contradiction is 'the most certain of all principles' (Metaphysics 1005b22). But this is admittedly quite speculative - we ought to be allowed to question even the 'first' principles.

It would certainly be enough to challenge the metaphysical necessity of LNC, or other logical principles, if empirical evidence to the effect that the principle is not true of every situation or every object and property would be found. ${ }^{15}$ This is what Priest has attempted to show with the case of change and Zeno's paradoxes, but I remain unconvinced. As I have argued (Tahko 2009), Priest's examples can all be accounted for in terms of semantic rather than metaphysical dialetheism - a distinction developed by Edwin Mares (2004). The idea is that there may be indeterminacy in semantics, but this does not imply that there is

15 I have in mind concrete objects in the first place; see Estrada-González (2013) for a case to the effect that there are abstracta which violate LNC in this sense. 
indeterminacy in the world. Only the latter type of indeterminacy would corroborate the existence of a genuinely possible paraconsistent configuration of the world. Since I have not seen a convincing case to the effect that such a configuration is genuinely possible, I take it that LNC is a good candidate for a metaphysically necessary principle. If I am right, this means that a paraconsistent possible world could not have turned out to accurately represent the actual world. The fact that there are paraconsistent models has no direct bearing on this question. I do not claim to have settled the status of LNC once and for all, but I think that a strong empirical case for the truth of LNC can be made, on the basis of the necessary constraints for the forming of a stable macrophysical world, i.e., the emergence of stable macrophysical objects.

I have developed the preceding line of thought before with regard to the Pauli Exclusion Principle (PEP) (Tahko 2012), and electric charge (Tahko 2009). For instance, as PEP states, it is impossible for two electrons (or other fermions) in a closed system to occupy the same quantum state at the same time. This is an important constraint, as it is responsible for keeping atoms from collapsing. It is sometimes said that PEP is responsible for the spaceoccupying behavior of matter - electrons must occupy successively higher orbitals to prevent a shared quantum state, hence not all electrons can collapse to the lowest orbital. Here we have a principle which captures a crucial constraint for any genuinely possible configuration of the world that contains macroscopic objects. Whether or not there are genuinely possible configurations that do not conform to PEP is an open question, but it seems unlikely that such a configuration could include stable macroscopic objects.

Consider the form of PEP: it states that two objects of a certain kind cannot have the same property (quantum state) in the same respect (in a closed system) at the same time. Compare this with Aristotle's formulation of LNC: 'the same attribute cannot at the same time belong 
and not belong to the same subject in the same respect' (Metaphysics 1005b19-20). LNC is of course a much more general criterion than PEP - it concerns one thing rather than things of a certain kind - but its underlying role is evident: if any fermion were able to both be and not be in a certain quantum state at the same time, then PEP would be violated and macroscopic objects would collapse. If LNC is needed to undergird PEP, then we have a strong case in favor of the metaphysical interpretation of LNC in worlds that contain macrophysical objects, given the necessity of PEP for the forming of macrophysical objects. This is of course not sufficient to establish the metaphysical necessity of either principle, but it is an interesting result in its own regard.

\section{Pluralism about logical truth}

Now that we have a rough idea about the metaphysical interpretation of logical truth, we can consider the implications of this interpretation in a wider context. Here I would like to focus on the topic of logical pluralism, which has lately received an increasing amount of attention. Perhaps the most influential form of logical pluralism derives from pluralism about logical consequence, i.e., the view that there are models in which the logical consequence relation is different, and irreconcilably so. Beall and Restall have formulated and defended this type of pluralism:

Given the logical consequence relation defined on the class of $\operatorname{cases}_{\mathrm{x}}$, the $\operatorname{logical}_{\mathrm{x}}$ truths are those that are true in all casesx. If you like, they are the sentences that are $\mathrm{x}$ consequences of the empty set of premises. The logical ${ }_{x}$ truths are those whose truth is yielded by the class of cases $x$ alone. Since we are pluralists about classes of cases, we 
are pluralists about logical truth. (Beall and Restall 2006: 100.)

If this is indeed what pluralism about logical truth amounts to, then it appears that anyone who accepts multiple classes of cases is a pluralist about logical truth. But what does 'being true in a case' mean? On the face of it, one might think that it means exactly the same as 'being true in a model', that is, we are talking about a type of relative truth familiar from Davidson. This would imply that anyone who accepts multiple classes of models will also be a pluralist about logical truth. Pluralism about logical truth would then mean only that there are multiple models, and we can talk about logical truth separately in each one of these models. But this would be a rather uninteresting sense of logical pluralism, at least from the point of view of the metaphysical interpretation of logical truth. However, as Hartry Field has recently pointed out, this cannot be what Beall and Restall have in mind. Moreover, Field suggests two reasons why model-theoretic accounts are irrelevant to logical pluralism:

One of these reasons is that by varying the definition of 'model', this approach defines a large family of notions, 'classically valid', 'intuitionistically valid', and so on; one needn’t accept the logic to accept the notion of validity. A classical logician and an intuitionist can agree on the model-theoretic definitions of classical validity and of intuitionist validity; what they disagree on is the question of which one coincides with genuine validity. For this question to be intelligible, they must have a handle on the idea of genuine validity independent of the model-theoretic definition. Of course, a pluralist will contest the idea of a single notion of genuine validity, and perhaps contend that the classical logician and the intuitionist shouldn't be arguing. But logical pluralism is certainly not an entirely trivial thesis, whereas it would be trivial 
to point out that by varying the definition of model one can get classical validity, intuitionist validity, and a whole variety of other such notions. (Field 2009: 348.)

And the second reason:

[I]f we were to understand 'cases' as models, then there would be no case corresponding to the actual world. There is no obvious reason why a sentence couldn't be true in all models and yet not true in the real world.

This connects up with the previous point: the intuitionist regards instances of excluded middle as true in all classical models, while doubting that they are true in the real world. (Field 2009: 348; italics original.)

Field goes on to suggest that Beall and Restall must have meant that there is an implicit requirement for interpreting 'truth in a case', namely, that truth in all cases implies truth. Field then argues that this will not produce an interesting sort of logical pluralism as the pluralist notion of logical consequence suggested by Beall and Restall does not capture the normal meaning of 'logical consequence'. But it should be noted that Beall and Restall (2006: 36 ff.) do say something about the matter. Specifically, they suggest that on one reading of 'case' (the TM account), Tarskian models are to be understood as cases. Another reading (the NTP or necessary truth-preservation account) takes possible worlds to be cases. Beall and Restall (2006: 40) add that the existence of a possible world that invalidates an argument entails the existence of an actual (abstract) model that invalidates the argument.

So, it is not clear that Field's critique is accurate, as Beall and Restall do suggest that there is a case that corresponds with the actual world - on the TM account it is a Tarskian model 
and on the NTP account it is a possible world. The latter is of immediate interest to us, given that the metaphysical interpretation of logical truth also makes use of the possible worlds jargon. Yet, Beall and Restall do not provide an interpretation of possible worlds, so it is not quite clear what the connection, if any, between the NTP account and the metaphysical interpretation of logical truth is.

Connecting all this with the analysis provided in the previous section, one might suggest that classes of cases are sets of metaphysically possible worlds, distinguished in terms of logical truths that are true in each set of possible worlds. Only one possible world is actual, but the logical truths that are true in the actual world will also be true in all worlds which are in the same set of possible worlds, i.e., these worlds may differ in other regards, but they are close to the actual world in the sense that all the logical truths are shared.

Accordingly, pluralists about logical truth, in the metaphysical sense, hold that there are distinct sets of possible worlds in which different logical truths hold. The metaphysical interpretation of logical truth can accommodate this sense of logical pluralism, provided that possible worlds are interpreted appropriately - this also enables us to preserve CI. ${ }^{16}$ However, accommodating pluralism in the metaphysical interpretation of logical truth does require a revision in our original definition (ML), which defined a sentence as logically true if and only if it is true in every genuinely possible configuration of the world. Since in this view of logical pluralism there can be proper subsets of genuinely possible configurations with different laws of logic, we must revise ML as follows:

16 Why is interpreting logical truth on the basis of metaphysical possibility the only way to preserve CI? Because we've seen that only by restricting our attention to metaphysically possible worlds can we preserve a sense of correspondence between logical truth and genuinely possible configurations of the world. Only metaphysically possible worlds are sufficiently constrained to take into account all the governing principles such as metaphysical a posteriori necessities. 
(ML-P) A sentence is logically true if and only if it is true in every possible world of a given subset of possible worlds representing genuinely possible configurations of the world.

ML-P can of course also accommodate the situation where the laws of logic are the same across all subsets of genuinely possible configurations, i.e., logical monism - in that case the relevant subset of possible worlds would not be a proper subset of the genuinely possible configurations.

An alternative formulation of ML-P is possible, dismissing subsets altogether. We could understand logical pluralism by giving different interpretations to 'genuinely possible configurations'. ${ }^{17}$ This formulation could be developed by adopting a line of thought from Gillian Russell (2008). Russell suggests that we can distill a sense of pluralism by understanding logical validity as the idea that in every possible situation in which all the premises are true, the conclusion is true (2008: 594), where possibility is ambiguous between logical, conceptual, nomological, metaphysical, or other senses of modality, hence producing a similar ambiguity concerning validity. A friend of the metaphysical interpretation of logical truth could accept this idea, but only provided that we prioritize the reading where possible situations reflect metaphysical possibility, as CI is preserved only in this reading. Nevertheless, there may still be room for a type of pluralism concerning metaphysical possibility and hence genuinely possible configurations. Unfortunately I have no space to develop this approach further.

It may be noted that since I have been discussing logical pluralism only with regard to the law of non-contradiction, the resulting sense of pluralism is limited. Given that I consider

17 Thanks to Jesse Mulder for suggesting this type of formulation. 
there to be strong reasons to think that LNC holds in the actual world, we can define a set of possible worlds in which the law of non-contraction holds, call it $W_{L N C}$. The assumption is that $W_{L N C}$ includes the actual world. But since I have made no mention of any other laws of logic that hold (in the metaphysical sense) in $W_{L N C}$, the sense in which we can talk of a logic may be questioned. In other words, it may be wondered if the resulting sense of logical pluralism is able to support a rich enough set of logical laws to constitute a logic. However, I suspect that the case can be extended beyond LNC. That is, we can extend the metaphysical interpretation to other laws of logic as well in such a way that a subset of $W_{L N C}$ may be defined. This is not quite as straightforward in other cases though.

Very briefly, consider modus ponens $(A \wedge(A \rightarrow B)) \rightarrow B)$. If thought of as a rule, it is not obvious that modus ponens can be applied to the world in the sense that I have suggested with regard to LNC. Yet, there are clear cases of physical phenomena that feature a modus ponens type structure. As a first pass, causation might be offered as a candidate of 'real world modus ponens', but there are obvious complications with this suggestion, as it depends on one's theory of causation. However, there are better candidates. Take the simple case of an electron pair in a closed system, where two electrons occupy the same orbital. As we've already observed, two electrons in a closed system are governed by the Pauli Exclusion Principle. In particular, since the electrons cannot be in the same quantum state at the same time, we know that the only way for them to occupy the same orbital (i.e., having the same orbital quantum numbers) is for them to differ in spin (i.e., to have different spin quantum numbers). Accordingly, when we observe electron A having spin-up, we immediately know that any electron, $B$, on the same orbital as $A$ must have spin-down. Moreover, there can be only two electrons on the same orbital and they must always have opposite spin.

If cases such as the one for a 'real world modus ponens' can be found, then we may indeed 
have a rich enough set of logical laws to constitute a logic, enabling the suggested interpretation of logical pluralism. The resulting subset could be called $W_{L N C+M P}$.

This hardly exhausts the debate about logical pluralism, but it appears that there are ways, perhaps several ways, to accommodate pluralism about logical truth within the metaphysical interpretation.

\section{Conclusion}

In conclusion, I have demonstrated that there is a coherent metaphysical interpretation of logical truth, and that this interpretation has some interesting uses, such as applications regarding logical pluralism. It has not been my aim to establish that this interpretation of logical truth is the correct one, but only that it is of special interest to metaphysicians. I have assumed rather than argued for a type of realism about logic for the purposes of this investigation, but I contend that for realists about logic, one interesting interpretation of logical truth is the one sketched here. ${ }^{18}$

18 Thanks to audiences at the University of Tampere Research Seminar and the First Helsinki-Tartu Workshop in Theoretical Philosophy, where earlier versions of the paper were presented were presented. In particular, I'd like to thank Luis Estrada-González for extensive comments. In addition, I appreciate helpful comments from Franz Berto and Jesse Mulder. Thanks also to Penny Rush for editorial comments. The research for this article was made possible by a grant from the Academy of Finland. 